\title{
Characterization of GEKKO/HIPER-Driven Shock Waves for Equation-of-State Experiments in Ultra-High-Pressure Regime
}

\author{
OZAKI Norimasa ${ }^{1,3}$, TANAKA Kazuo A., ${ }^{2,3}$, ONO Takatoshi ${ }^{2,3}$, TAKAMATSU Kikuo ${ }^{2,3}$, NAGAI Keiji ${ }^{3}$, \\ SHIGEMORI Keisuke ${ }^{3}$, NAKAI Mitsuo ${ }^{3}$, MIYANAGA Noriaki ${ }^{2}$, AZECHI Hiroshi ${ }^{3}$ and YAMANAKA Tatsuhiko ${ }^{3}$ \\ ${ }^{1}$ Laboratoire pour l'Utilisation de Lasers Intenses, Ecole Polytechnique, 91128 Palaiseau Cedex, France \\ ${ }^{2}$ Faculity of Engineering, Osaka University, Suita, Osaka 565-0871, Japan \\ ${ }^{3}$ Institute of Laser Engineering, Osaka University, Suita, Osaka 565-0871, Japan \\ (Recieced 12 February 2004 / Accepted 10 May 2004)
}

\begin{abstract}
The GEKKO/HIPER-laser driven shock experiments were characterized in detail for studies on equation-ofstate (EOS) in ultra-high pressure regime. High-quality shock waves were produced with optically smoothed laser beams. Key issues on EOS measurement with shock waves, the spatial uniformity and the temporal steadiness of shock, and the preheating problem were investigated by measurements of the self-emission and reflectivity from target rear surface. Our experiments and analysis based on impedance matching method were validated by use of double-step targets consisting of two Hugoniot standard metals. Extreme shock waves previously only achieved in nuclear explosion experiments were generated using the laser direct-drive experimental scheme.
\end{abstract}

\section{Keywords:}

equation of state, Hugoniot, shock wave, high pressure, high power laser

\section{Introduction}

Equation-of-state (EOS) data of materials in high pressure regime provide essential information for high-energydensity physics including astrophy [1,2], geophysics [3,4], and inertial fusion energy (IFE) research [5,7]. For example in the IFE researches, compression efficiency and shock structure in fusion capsules critically depend on the EOS [5,6]. Such hydrodynamic and thermodynamic conditions are evaluated by numerical codes generally employing wellknown EOS models [8,9]. In order to design and address any experiments, accurate EOSs of materials are required.

Tera-Pascal $(\mathrm{TPa},=10 \mathrm{Mbar})$ pressures can be achieved only by strong-shock waves driven with high-energy pulse powers such as nuclear explosions [10-14]. Although highpower laser facilities have demonstrated the potential of investigating the EOS at the ultra-high pressures, Hugoniot data above $1 \mathrm{TPa}$ have been limited to a few cases [15-23]. Additionally, data for low-Z materials with relatively good accuracy are available in only a few publications [19,20,23].

In order to obtain accurate Hugoniot data, high-quality shocks with sufficient spatial uniformity (planarity) and temporal steadiness have to be established [15,24]. The laser direct-drive technique has advantages that enable control of shock pressure profile, high-energy conversion efficiency, and very simple experimental geometry. On the other hand, the preheating effect remains a disadvantage. It is well known that the irradiation by optically smoothed laser beams at a short laser-wavelength is effective to restrain preheating due to suprathermal electrons. At laser intensities up to a few $10^{14}$ $\mathrm{W} / \mathrm{cm}^{2}$, preheating is mainly due to thermal $\mathrm{x}$-ray radiation. This type of preheating can be suppressed by use of low-Z ablator and/or thick piston layers [25]. Once the preheating suppressed which may be verified with a temperature measurement, shock velocity can be measured with good accuracy in laser indirect-drive experiments [17] and reliable Hugoniot points can be determined by using the impedance matching method (IMM) [26]. The IMM experiments derive EOS data for a sample relative to the EOS of a standard material, called as relative EOS experiments. Laser-driven absolute EOS measurements require very high-power laser with long pulse duration to generate a main shock loading and a strong backlighter x-ray pulse [20,27,28]. Moreover, the alignment relation between the target and the diagnostic (x-ray backlight) is difficult because any tilt of the plane target affects the observed compressibility [29]. Thus, experiments based on IMM are often preferred.

In this paper, we present experiments to characterize laser-driven shock wave property for the relative EOS measurements with the GEKKO/HIPER (High Intensity Plasma Experimental Research) system at the Institute of 
Laser Engineering (ILE), Osaka University. The spatial and temporal uniformities of shock waves are verified by measuring self-emission and the probe-light reflection from the target rear side. The preheating is evaluated by the rearemission and the reflectivity measurements. The validity of our experiments based on IMM is confirmed with double step targets consisting of aluminum $(\mathrm{Al})$ and copper $(\mathrm{Cu})$ : wellknown Hugoniot standard metals. The present experiments show that EOS data at ultra-high pressures explored previously in nuclear explosion experiments are accessible for any material.

\section{Experimental Conditions}

A series of experiments was conducted using the HIPER laser facility [30] which is an irradiation system on the GEKKO XII (GXII): Nd glass laser system at the ILE [31]. The facility provides one-dimensional compression by smoothed laser beams with short wavelength and high intensity. In the system, twelve beams of the GXII are bundled in an $F / 3$ cone angle. The wavelength of the nine beams is $351 \mathrm{~nm}(3 \omega)$ that is the third harmonic of a $1053 \mathrm{~nm}(\omega)$ fundamental. In the $3 \omega$ beams, smoothing by the spectral dispersion (SSD) technique [32] was applied. Kinoform phase plates (KPPs) [33] were installed for all the beams to obtain a uniform irradiation pattern. The temporal behavior of the laser pulse was approximately a square shape in time, in order to generate a well-defined shock pulse, with a full width at half maximum (FWHM) of $2.5 \mathrm{~ns}$ and a rise and fall time of 100 ps. The focal-spot diameter was typically $600 \mu \mathrm{m}$. The laser irradiation spots were routinely monitored by timeresolved and time-integrated x-ray cameras.

A schematic view of the experimental configuration is shown in Fig. 1. Three diagnostic systems were used to measure a target rear-side event at the same time. The first is a measurement of self-emission from shock breakout at the rear surface using a visible streak camera (streak camera 1 in Fig. 1). The self-emission signals were collected by an $F / 2.8$ lens and were image-relayed on the slit of the streak camera by a microscopic-objective and achromatic lenses. The target rear image was rotated vertically by a dove prism to arrange the steps edge of target on the streak slit. In this optical path, band-cut filters were inserted for the $2 \omega$ and $3 \omega$ of the GXII wavelength.

The second diagnostic system was a measurement of the reflection of a probe laser from the target rear side. An injection-seeded Q-switched Nd: YAG (Yttrium Aluminum Garnet) laser was used as the probe light. The maximum energy was $0.7 \mathrm{~J}$ at a wavelength of $532 \mathrm{~nm}$. The original pulse duration was approximately $8 \mathrm{~ns}$ (FWHM) with a Gaussian shape. The probe laser was injected into one end of an optical fiber by a lens and was passed through the fiber to near the vacuum target chamber. Another end of the fiber was coupled with a fiber collimator and a lens, thus the probe light was collimated in front of a focal lens of $F / 3$ in the backlighter beam line of the HIPER system. The YAG probe illuminated the target rear surface using the focal lens of the backlighter beam. Specular reflection of the probe was collected by the same optics utilized in the self-emission measurement. The light was reflected by a laser mirror at the probe wavelength of $532 \mathrm{~nm}$ and was focused on the slit of another streak camera system (streak 2 in Fig. 1). Note that a notch filter (less than $10 \mathrm{~nm}$ bandwidth) for the $532 \mathrm{~nm}$ of YAG was installed to prevent the self-emission from the probe light signal.

The third diagnostic was a radiation pyrometer based on a color temperature measurement. This system allowed us to obtain spatially-, temporally-, and spectrally-resolved images using one streak camera (streak 3 in Fig. 1) [34]. We could obtain two different spectral images onto the streak slit by using a biprism and two different colored filters. In order to increase accuracy and sensitivity for color temperature measurements, we choose the spectrum regions of blue (385$469 \mathrm{~nm}$ ) and ultra-violet $(284-327 \mathrm{~nm})$. The details of this measurement is described by Ono et al. in this issue.

In our impedance-matching experiments, $\mathrm{Al}$ is used as EOS standard material. Target structure in irradiation side by the HIPER is classified into two types. First is a plane Al base target for low laser intensity. Second is a two-layered base target with a $\mathrm{CH}$ (polystyrene) ablator to suppress thermal radiation preheating in high laser intensity. The $\mathrm{CH}$ is overcoated with a thin Al layer of 1,000 $\AA$ in thickness that prevents direct laser shine-through in the $\mathrm{CH}$. Using a numerical code MY1DL based on a 1D hydrodynamic Lagrangian scheme [35], we optimized the target thicknesses to maintain steady-shock pressures under our laser conditions. Typically the optimized thicknesses of $\mathrm{Al}$ was between 30 and $70 \mu \mathrm{m}$. To fabricate step targets, an adhesion technique was used with a single molecular membrane coating [36,37]. All targets were characterized by a confocal laser 2D-scanning microscope with a minimum scale value in height of $10 \mathrm{~nm}$. Portions of those, in particular EOS measurement targets, were characterized within the area of the HIPER irradiation spot by a 1D-scanning microscope with a height resolution

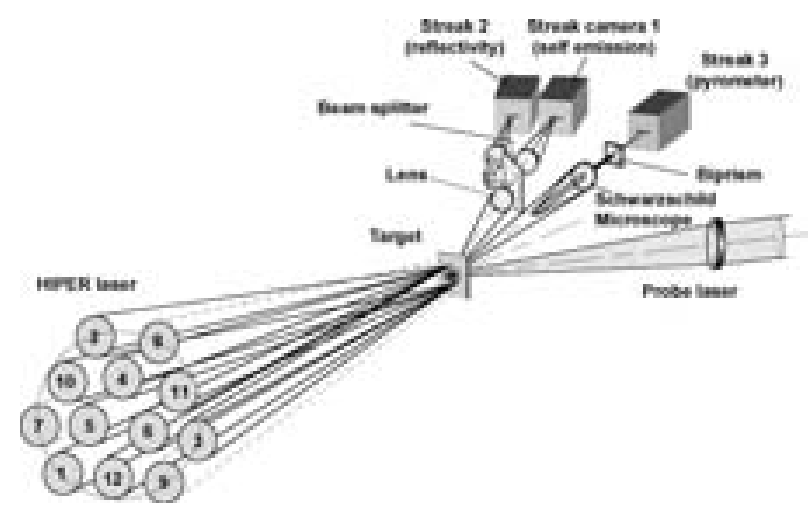

Fig. 1 Experimental schematic. Three streak cameras were used to observe a target rear event at the same time. The number 7, 8 and 9 beam are PCL [44], and the others are SSD [32] beams. 
of $1 \mathrm{~nm}$.

\section{Shock Uniformity}

In the measurements of Hugoniot EOS, high-quality planar shocks are required. The spatial uniformity was verified in the present experiments with planar Al targets with 40 to $100 \mu \mathrm{m}$ thicknesses. Figure 2 shows the typical streaked image of self-emission from the target rear surface at a laser intensity of $9.2 \times 10^{13} \mathrm{~W} / \mathrm{cm}^{2}$ and a thickness of $40 \mu \mathrm{m}$. Time proceeds from the top to the bottom. The central flat region of shock wave was estimated as over $230 \mu \mathrm{m}$ diameter, with which a variation in breakout time of \pm 8 ps. The shock arrival time was defined as the leading edge mesial point of the shock emission signal. The flat region was sufficiently wide for EOS experiments with several percent of accuracy. Here, since the order of the shock velocity can be considered as several $10 \mathrm{~km} / \mathrm{s}$ throughout the experimental campaign, the shock wave can travel a distance of $100 \mathrm{~nm}$ order in the $8 \mathrm{ps}$. This distance is comparable to the surface roughness of the Al. Of course, The variation includes the effect of attenuation due to two dimensional effects, particularly in the left and right hand side from the center of the shock wave.

The shock-condition generated on the Hugoniot has to be in equilibrium behind the shock wave discontinuity, and has to be temporally steady for a duration long enough for the observations. The temporal uniformity of the HIPERdriven shock velocity was measured with a wedged target to confirm the steadiness of the shock pressure; the shock breakout time should be proportional to distance along the incline. The wedged targets with an angle of $9.4^{\circ}$ were made by a precision machining technology. Figure 3 is a raw streaked image of the rear self-emission using a wedged target (the target structure is also shown). The shock-breakout time was delayed linearly in distance along the incline to \pm 18 ps root-mean-square (RMS) in around $2 \mathrm{~ns}$ duration. The temporal uniformity of the shock wave was hence estimated as $\pm 1.13 \%$. In typical EOS-targets the thickness region of 40 $60 \mu \mathrm{m}$ was used in our experiments for the fair steadiness.

The shape of drive-laser pulse strongly contributes to that of the generated shock pulse. This is advantageous in direct laser-drive technique for easily controlling the pulse shape. The inset in Fig. 3 shows an example of the pulse shape provided by the laser system in the EOS experimental campaign. The laser pulse was nearly flat-topped with an intensity fluctuation in the saturated zone of less than $\pm 1.4 \%$ over $1.8 \mathrm{~ns}$.

\section{Preheating}

$\mathrm{X}$ rays and superthermal electrons are emitted from laserablation plasma. These radiations can heat the ambient matters of the cold initial state before the shock front overtakes. The target condition before the shock-front arrival contributes to the final shocked state on the Hugoniot. If the preheating effect significantly changes the initial condition of the sample to a new and unknown state, this effect can consequently obscure or ruin the obtained Hugoniot data. We characterized

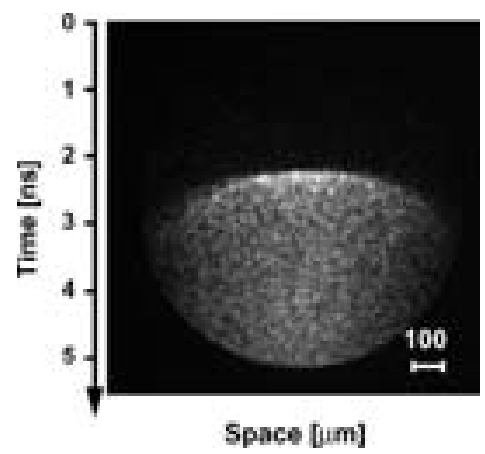

Fig. 2 Typical raw streaked image by self-emission measurement with Al planar target of $40 \mu \mathrm{m}$ thick-ness.

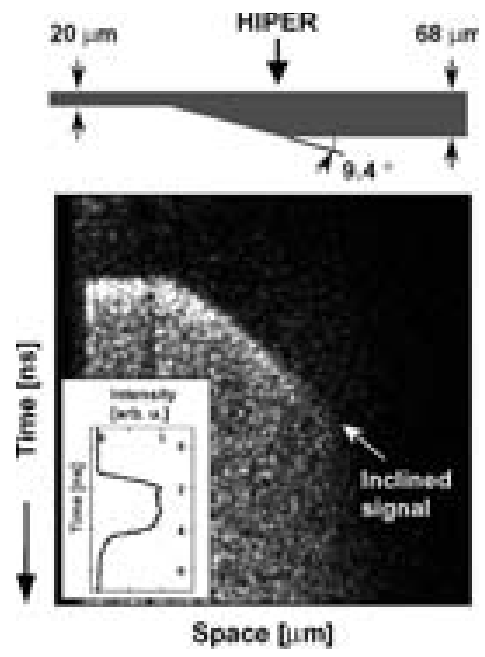

Fig. 3 Typical streaked image by self-emission measurement with a wedged Al target. The inset is temporal pulse shape of the drive laser.

the preheating temperature level in our experimental conditions. In these experiments, preheating is mainly due to $\mathrm{x}$ rays from the critical density plasma because laser intensity is well below the threshold for any nonlinear parametric instability inducing suprathermal-electron preheating. Moreover, our laser wavelength and uniform irradiation should be effective in reducing the instabilities [25].

Figure 4(a) shows the temporal history of the rear emission from a planar Al target represented in Fig. 2. This profile is a typical signal from strong-shock breakout, and shows that a well-defined shock is generated. Taking into account the time resolution of the diagnostic system, the measured rise time is shorter than $20 \mathrm{ps}$. After shock passage, the rapid decay denotes that the plasma is cooled due to the expansion into the vacuum without heating by $\mathrm{x}$ rays from the HIPER irradiation side. No significant emission is detected before the shock emergence at the free surface. We therefore believe that the preheating level is less than the detection limit of this measurement, a $0.9 \mathrm{eV}$ blackbody temperature.

The reflected probe-light measurement at the target rear- 
surface is more sensitive to the preheating effect, investigated by Bennuzi et al. for the first time [25]. The reflectivity is defined as the ratio of reflected light intensity of the shocked target to incident light intensity. An example of reflectivity for a $40 \mu \mathrm{m}$-thick $\mathrm{Al}$ is shown as a function of time in Fig. 4(b). The lower solid thin and grey thick curve indicate the intensity profile of reflected and incident light, respectively, and the upper dotted curve shows the calculated reflectivity. The horizontal bar in the reflectivity curve is a guide to the eye, which denotes $100 \%$ reflection. The drive-laser irradiance was $5.0 \times 10^{13} \mathrm{~W} / \mathrm{cm}^{2}$. Shock breakout time is shown as an arrow. We find that the reflectivity decreases rapidly at shock emergence. No significant decrease of reflectivity was detected before the shock arrival at the rear surface. The decrement before the shock breakout can be estimated as not more than $7 \%$. This is consistent with about $0.08 \mathrm{eV}$ preheating level [25].

Finally, for this temperature level resulting from thermal radiation, as the increase of the shock propagating distance due to free surface expansion and of the shock velocity due to temperature increment at a preheated region can be comparable, the competition between these factors can make shock velocity measurements insensitive to the radiation preheating [38].

Assuming that the corresponding expansion velocity of Al free surface is $0.3 \mathrm{~km} / \mathrm{s}$ at the detection limit level of preheating temperature, we can expect that the decrease of density in $40 \mu \mathrm{m} \mathrm{Al}$ base plate is approximately less than $0.9 \%$ and that in the step is much less than it through this experimental campaign. This value was applied to the error analysis. This amount of preheating temperature is probably overestimate for unknown and Al step in double-step target.

\section{Hugoniot Measurement for Copper}

Our laser-driven IMM experiments were validated with using double-step target consisting of two Hugoniot standards, $\mathrm{Al}$ and $\mathrm{Cu}$ [39]. Figure 5 is an example of typical image obtained by the reflected light measurement (the target structure is also shown). The $\mathrm{Al}$ and $\mathrm{Cu}$ step are on the rightand left-hand sides, respectively. The time intervals, $t^{\mathrm{Al}}$ and $t^{\mathrm{Cu}}$, correspond to the transit time of the shock propagating through each step, where superscripts denote the material. As the step heights were known, the shock velocity of Al, $U_{\mathrm{s}}^{\mathrm{Al}}$, was $26.43 \pm 0.45 \mathrm{~km} / \mathrm{s}$ and that of $\mathrm{Cu}, U_{\mathrm{s}}^{\mathrm{Cu}}$, was $19.47 \pm 0.29$ $\mathrm{km} / \mathrm{s}$.

The Al Hugoniot has been experimentally and theoretically investigated over a wide range of pressures. Here, we used the SESAME model [9] of Al to determine the final Hugoniot points. The model is in good agreement with experimental Hugoniot data up to approximately $2 \mathrm{TPa}$.

The $u_{\mathrm{p}}^{\mathrm{Al}}$ and pressure $P^{\mathrm{Al}}$ state corresponding to the measured shock velocity of $26.43 \mathrm{~km} / \mathrm{s}$ were determined as $16.29 \mathrm{~km} / \mathrm{s}$ and $1.16 \mathrm{TPa}$, respectively. When the shock wave propagates through the interface between $\mathrm{Al}$ and $\mathrm{Cu}$, a reflected shock travels in the primary shocked Al. The reflected shock condition is given by the intersection of the

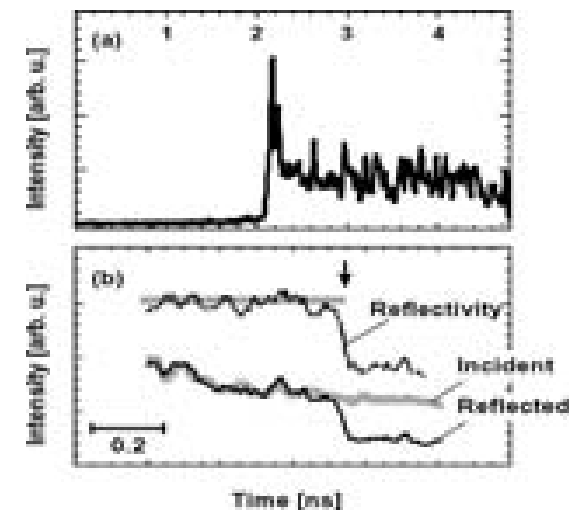

Fig. 4 (a) Typical temporal profile of the rear emission at a 40 $\mu \mathrm{m}$-thickness of Al. The drive laser irradiance is $9.2 \times 10^{13}$ $\mathrm{W} / \mathrm{cm}^{2}$. (b) Typical reflectivity signal, and the intensity profile of reflected and incident probe light to determine the reflectivity. Horizontal grey line shows $100 \%$ reflection. Shock arrival time is indicated by an arrow.
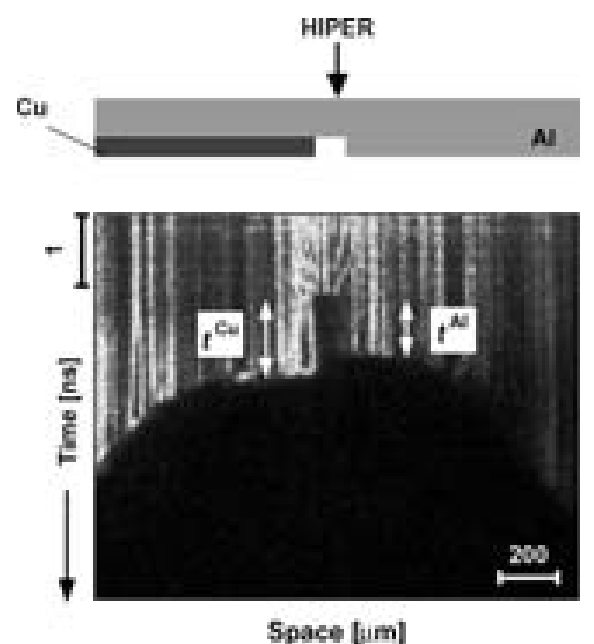

Fig. 5 Streaked image with double standard target by reflected light measurement. The step height of $\mathrm{Al}$ and $\mathrm{Cu}$ are 19.68 and $19.79 \mu \mathrm{m}$, respectively. The $t^{\mathrm{Al}}$ and $t^{\mathrm{Cu}}$ indicate transit time for the shock traveling through each step, respectively.

re-shock curve of $\mathrm{Al}$ and the Rayleigh line of $\mathrm{Cu} ; P=$ $\rho_{0}^{\mathrm{Cu}} U_{\mathrm{s}}^{\mathrm{Cu}} u_{\mathrm{p}}$ in $P-u_{\mathrm{p}}$ plane. The reflected shock curve of $\mathrm{Al}$ was calculated based on the SESAME database. Consequently, the particle velocity in $\mathrm{Cu}$ was $11.31 \mathrm{~km} / \mathrm{s}$, and the pressure was $1.97 \mathrm{TPa}$. These values had accuracies better than $3.1 \%$. The determination manner of these errors is described in Ref 40 [40].

The Hugoniot data of $\mathrm{Cu}$ and $\mathrm{Al}$ are plotted in Fig. 6. Circles denote $\mathrm{Cu}$ Hugoniot points. The solid symbol indicates present work. The open circles are the results from published papers with gas guns [41] and nuclear explosions [14]. Three $\mathrm{Cu}$ data points from the highest pressure shown are the results from IMM experiments using nuclear explosions, and others are from absolute experiments using 
gas guns. Grey circles are several examples by Rothman $e t$ $a l$. in indirectly-driven laser experiments with hohlra [17,42]. The solid curve shows the SESAME Hugoniot EOS for $\mathrm{Cu}$ (SESAME 3332). Our result is fully consistent with this model and previous works using different techniques.

\section{Intense Shocks in Aluminum}

Figure 7 shows the typical streaked image of selfemission from the rear surface of an $\mathrm{Al}$ stepped target. The step height was $20 \mu \mathrm{m}$, the shock velocity being $37.9 \mathrm{~km} / \mathrm{s}$. According to the SESAME database, the corresponding Hugoniot pressure and density of the shocked Al are calculated as $2.52 \mathrm{TPa}$ and $8.80 \mathrm{~g} / \mathrm{cm}^{2}$, respectively.

The results are summarized in Fig. 8. Solid circles and Open diamonds indicate experimental data from the present and previously published works [13,39], respectively. Dotted curve shows theoretical Hugoniot calculated by the SESAME model (SESAME 3718) [9]. All referenced plots in the TPa region were obtained from nuclear explosion experiments. The present ablation pressures, $P_{\mathrm{abl}}$ in $\mathrm{Al}$ demonstrate that the GEKKO/HIPER-driven EOS experimental system of direct drive scheme can provide ultra-intense shock pressure, previously explored only in nuclear explosion experiments for several materials with different initial densities [43]. This suggests us to obtain new EOS data for many materials in ultra-high pressure regime (>10 $\mathrm{TPa})$.

\section{Conclusions and Summary}

In conclusion, we have presented experiments to characterize GEKKO/HIPER-driven shock waves for studies of EOS in ultra-high pressure regime. In order to generate high-quality shock waves in the laser direct-irradiation scheme, we used optically smoothed laser beams. The laser intensity was up to $10^{14} \mathrm{~W} / \mathrm{cm}^{2}$ or higher with a wavelength of $351 \mathrm{~nm}$ and a squared pulse of $2.5 \mathrm{~ns}$ duration.

The key issues for laser-driven EOS experiments were confirmed with planar and wedged Al targets and with selfemission and reflection measurements. Shock planarity was over $230 \mu \mathrm{m}$ in the central flat region, which is sufficient for the spatial scale of our EOS experiments. The shock pressure was typically $\pm 1.3 \%$ steady for approximately $2 \mathrm{~ns}$ corresponding to the shock propagating time between target thicknesses of 40 and $60 \mu \mathrm{m}$. Preheating effects were investigated from the temporal profile of the emission and reflectivity; significant evidence for preheating was not detected before shock arrivals at the target rear surface. The temperature level was predicted as not more than $0.08 \mathrm{eV}$ for intensities to approximately $7 \times 10^{13} \mathrm{~W} / \mathrm{cm}^{2}$. This indicated that radiation preheating did not strongly affect the shock velocity measurements.

We verified our experimental technique based on the impedance matching method using double-step target consisting of Hugoniot standard metals of $\mathrm{Al}$ and $\mathrm{Cu}$. The result was in agreement with previous experimental data by different tools and an EOS model. This assures the validity of EOS experiments for unknown materials.
Extremely fast shocks in Al standard were created with systematization and reproducibility. From the shock velocities,

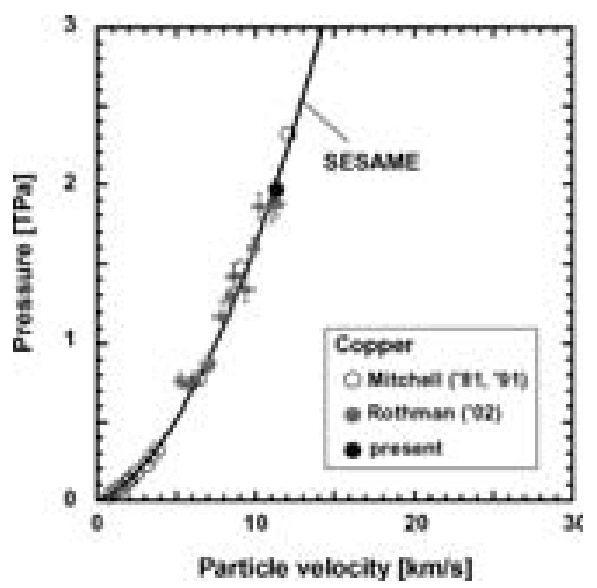

Fig. 6 Cu Hugoniot data. Solid circle is present data. Open circles are results with nuclear explosions and gas guns $[14,41]$. Grey circles are Cu data driven by laser [17]

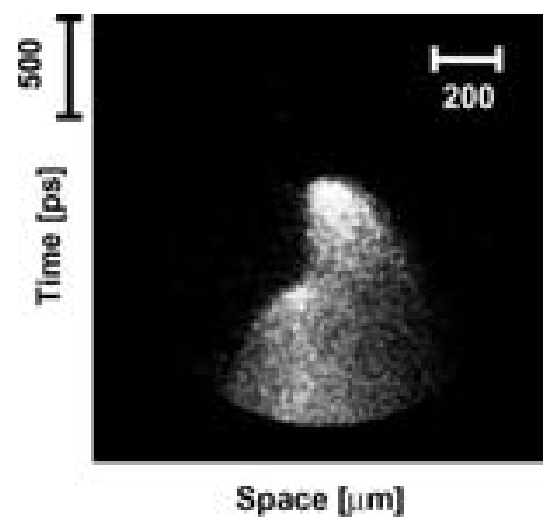

Fig. 7 Typical streaked image with Al stepped target by selfemission measurement.

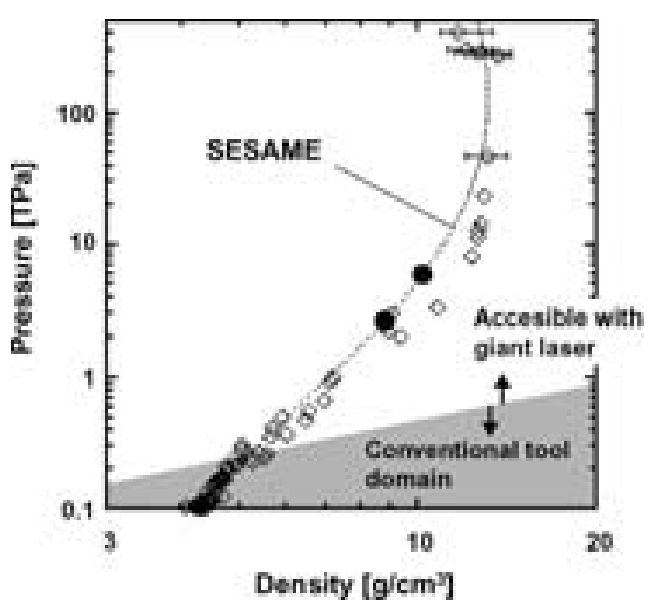

Fig. 8 Summarized Al high-pressure data. Solid circles and open diamonds are experimental data from the present and previously published works [13,39], respectively. Dotted curve indicates theoretical Hugoniot calculated by the SESAME model (SESAME 3718) [9]. 
we could estimate the corresponding shock pressures $\left(=P_{\mathrm{abl}}\right)$ on Hugoniot as up to 5.8 TPa using SESAME table. These results indicate that the laser-directly-driven experiments can provide multi-TPa EOS data for arbitrary unknown materials previously accessible only in nuclear explosion experiments. Note that the presented data are not fairly-obtained Hugoniot points. To establish accurate EOS standard in multi-TPa regime, absolutely determination of Hugoniot for $\mathrm{Al}$ is required. Laser-driven absolute experiments were carried out only for optically thin material such as polystyrene, because, for example, very high-power lasers with long pulse duration were needed to generate a main shock loading and a strong backlighter x-ray pulse [20,27]. We will conduct novel absolute observation of Hugoniot variables for the important metal as Hugoniot standard in future experiments.

\section{Acknowledgments}

The authors gratefully acknowledge the valuable support for the experiments by the technical crews and scientists at the ILE. Two authors (T.O. and N.O.) would like to thank S. Fujioka, and Y. Tohyama and H. Nishimura (ILE) for preparing diagnostic instruments. The authors would like to thank M. Koenig (Ecole Polytechnique) for providing EOS data.

This work was performed under the auspices of the Japan Science and Technology Corporation (JST) by Osaka University under Contract No. A2-12308020.

\section{References}

[1] W.J. Nellis, M. Ross and N.C. Holmes, Science 269, 1249 (1995).

[2] W.J. Nellis, Planet. Space Sci. 48, 671 (2000).

[3] J.H. Nguyen and N.C. Holmes, Nature 427, 339 (2004).

[4] V.N. Zharkov, Phys. Earth Planet. Inter. 109, 1 (1998).

[5] S.W. Haan et al., Phys. Plasmas 2, 2480 (1995).

[6] J.D. Lindl, Phys. Plasmas 2, 3933 (1995).

[7] T.R. Dittrich et al., Phys. Plasmas 6, 2164 (1999).

[8] R.M. More et al., Phys. Fluids 31, 3059 (1988).

[9] SESAME, the LANL equation of state database, Los Alamos National Laboratory, LA-UR-92-3407 (1992). Copies may be ordered from the National Technical Information Service, Springfield, Virginia 22161.

[10] C.E. Ragan III, Phys. Rev. A 25, 3360 (1982).

[11] W.J. Nellis et al., J. Appl. Phys. 82, 2225 (1997).

[12] W.J. Nellis et al., Phys. Rev. Lett. 60, 1414 (1988).

[13] A. Vladimirov, JETP Lett. 39, 82 (1984).

[14] A.C. Mithcell et al., J. Appl. Phys. 69, 2981 (1991).

[15] M. Koenig et al., Phys. Rev. E 50, R3314 (1994).

[16] M. Koenig et al., Phys. Rev. Lett. 74, 2260 (1995).
[17] S.D. Rothman et al., Phys. Plasmas 9, 1721 (2002).

[18] A. Benuzzi et al., Phys. Rev. E 54, 2162 (1996).

[19] M. Koenig et al., Appl. Phys. Lett. 72, 1033 (1998).

[20] R. Cauble et al., Phys. Rev. Lett. 80, 1248 (1998).

[21] D. Batani et al., Phys. Rev. B 61, 9287 (2000).

[22] D. Batani et al., Phys. Rev. Lett. 88, 235502 (2002).

[23] D. Batani et al., Phys. Rev. Lett. 92, 065503 (2004).

[24] Th Löwer et al., Phys. Rev. Lett. 72, 3186 (1994).

[25] A. Benuzzi et al., Phys. Plasmas 5, 1 (1998).

[26] Y.B. Zel'dovich and Y.P. Raizer, Physics of Shock Waves and High-Temperature Hydrodynamic Phenomena (Academic Press, New York, 1966).

[27] L.B. Da Silva et al., Phys. Rev. Lett. 78, 483 (1997).

[28] Recently, very interesting absolute measurement has been performed with a relatively small laser driver and velocity interferometer diagnostic in sub-TPa pressure regime; A. Benuzzi-Mounaix et al., Phys. Plasmas 9, 2466 (2002).

[29] W.J. Nellis, Phys. Rev. Lett. 89, 165502 (2002).

[30] N. Miyanaga et al., in Proceedings of the 18th International Conference on Fusion Energy (IAEA, Sorrento, Italy, 2001), IAEA-CN-77.

[31] C. Yamanaka, in proceedings of the Inertial Fusion Sciences and Applications 99, Bordeaux, 1999, edited by C. Labaune, W.J. Hogan and K.A. Tanaka (Elsevier, Paris, 2000) p.19.

[32] S. Skupsky et al., J. Appl. Phys. 66, 3456 (1989).

[33] S.N. Dixit et al., Opt. Lett. 21, 1715 (1996).

[34] T.A. Hall et al., Phys. Rev. E 55, R6356 (1997).

[35] T. Kadono et al., J. Appl. Phys. 88, 2943 (2000).

[36] K. Nagai et al., Jpn J. Appl. Phys. 41, L1184 (2002).

[37] K. Takamatsu et al., Phys. Rev. E 67, 056406 (2003).

[38] J.J. Honrubia et al., J. Quant. Spect. Rad. Transfer. 61, 647 (1999).

[39] A.V. Bushman, I.V. Lomonosov and V.E. Fortov, Equations of State for Metals at High Energy Density (Inst. Chem. Phys., Chernogolovka, 1992) (in Russian).

[40] N. Ozaki et al., Phys. Plasmas 11, 1600 (2004).

[41] A.C. Mithcell and W.J. Nellis, J. Appl. Phys. 52, 3363 (1981).

[42] $\mathrm{Cu}$ Hugoniot data are available also from other laser experiments; Ref. 18 and A.M. Evans et al., Laser Part. Beams 14, 113 (1996).

[43] In few indirect-drive experiments, more extreme pressures $\left(\neq P_{\mathrm{abl}}\right)$ were reported. For example, Cauble $e t$ al. reached $75 \mathrm{TPa}$ for gold using a hahlraum technique coupled with flyer impact method; R. Cauble et al., Phys. Rev. Lett. 70, 2102 (1993).

[44] H. Nakano et al., J. Appl. Phys. 73, 2122 (1993). 